\title{
Monitoring Computer Activities with Cloud to Device Messaging (C2DM)
}

\author{
Suwitno ${ }^{1}$, Rino $^{2}$, \\ ${ }^{1}$ Universitas Buddhi Dharma, Manajemen Informatika, Banten, Indonesia \\ ${ }^{2}$ Universitas Buddhi Dharma, Sistem Informasi, Banten, Indonesia
}

\begin{tabular}{|c|c|}
\hline SUBMISSION TRACK & $\begin{array}{llllllll}A & B & S & T & R & A & C & T\end{array}$ \\
\hline $\begin{array}{l}\text { Received : February 1, } 2018 \\
\text { Final Revision: February 6, } 2018 \\
\text { Available Online: February 27, } 2018\end{array}$ & $\begin{array}{l}\text { In a particular division, a supervisor needs to perform } \\
\text { monitoring of the activities carried out at his employers to } \\
\text { improve the performance of the work of his subordinates. }\end{array}$ \\
\hline KEY WORD & $\begin{array}{l}\text { Leaders are often not in place to monitor the activities of his } \\
\text { subordinates in carrying out their responsibilities. One }\end{array}$ \\
\hline $\begin{array}{l}\text { Push Notification, Device Monitoring, Cloud to } \\
\text { Device Messaging (C2DM) }\end{array}$ & $\begin{array}{l}\text { solution to overcome these problems is to supervise the use of } \\
\text { their computer by means of push notification. By utilizing the }\end{array}$ \\
\hline CORRESPONDENCE & $\begin{array}{l}\text { technology of Cloud to Device Messaging (C2DM), } \\
\text { supervisor can monitor computer activities of his employers }\end{array}$ \\
\hline $\begin{array}{l}\text { Telepon: } 081311190089 \\
\text { E-mail: suwitno@ubd.ac.id }\end{array}$ & $\begin{array}{l}\text { anywhere. Given this research, is expected to help the } \\
\text { supervisor in monitoring his employers despite not being in } \\
\text { place. This may indirectly improve performance in a division. } \\
\text { For further research can be developed monitoring in a real } \\
\text { time. }\end{array}$ \\
\hline
\end{tabular}

\section{INTRODUCTION}

In a division both in education and offices, activity on each employee's computer needs to be considered by the division leaders, because if one of the employees do not carry out their responsibilities properly, then it will slow down the process of responsibilities assigned by the leadership so that it can degrade the performance of the good work of the employee or related divisions. The basic task of a leader is to monitor the performance of the employers held with the aim of knowing the development of the division handled. With human mobility is increasing fast, the leader must be able to monitor the situation regularly, wherever and whenever he wants. Unfortunately, a leader is not always in place to carry out their duties. It's difficult for the leaders to monitor their employers. A solution that can be done to overcome these problems is need an application that can be monitoring the activities of their employers through their computers. Several studies have been done related to monitoring activities. From these studies, application monitoring can be a solution to overcome the existing problems. This study monitor activities conducted via computer by push notification. So that the leaders can view the activity that is done, then it will use the cloud to device messaging. Android Cloud to Device Messaging (C2DM) is a service that helps developers sends data from servers to their applications on android devices. The service provides a simple, lightweight mechanism that servers can use 
to tell mobile applications to contact the server directly, to fetch updated application or user data. Given this research is expected to facilitate the leaders for monitoring computer activities on each employee anywhere and anytime.

\section{METHOD}

\section{Cloud to Device Messaging (C2DM)}

Cloud to Device Messaging (C2DM) was made available from Android 3.0, where the goal was to make it easier for mobile applications to sync data with servers. The technology is used in several standard Google applications including Gmail, Contacts and Calendar. When messages are received on the Android client, the system will wake up the application via an Intent broad-cast, and pass the message data [5]. The message limit is set to 1024 bytes and developers are encouraged to send short messages, essentially notifying the mobile application that updated information can be retrieved from the server. $\mathrm{C} 2 \mathrm{DM}$ is a free service, and the maximum number of messages that can be sent is approximately 200,000 per day, however this can be in-creased if there is a need for more resources.

Before you can use C2DM application must be registered in advance on Google's servers using the name of the application package includes and Google email is still active to be verified by the Google.

While the mechanism of sending messages through C2DM, first android app connect to
C2DM Fontend using the existing email verified to get registration id, each device will get the registration id that is different, but the registration id impermanent therefore the system will renew the registration id periodically. Furthermore android application must send the registration id stored on a web server for the purpose is as assumptions during transmission of data to avoid errors at the receiver. Sending a message from the web server to server C2DM starting from a web server login to a Google server through Client Login Service and if successful will get the auth token while unique, auth token and registration id saved is included at the time of sending a message from the web server to C2DM Frontend. Demand is already authenticated will then be incorporated into C2DM Message Queue that will be forwarded to mobile applications using TCP / IP connection.

The concept is to build three applications that support the server side and client side. Applications that run on the server side in the form of a web server and a web service that hold the data $\log$ or history and liaison between the client-side applications. While the application consists of a client-side application that is installed keylogger on the computer that will be in reconnaissance activities and mobile applications used for android smartphone as a media receiver log information. The following figure 1 illustrates the push notification system architecture Cloud to Device Messaging (C2DM)

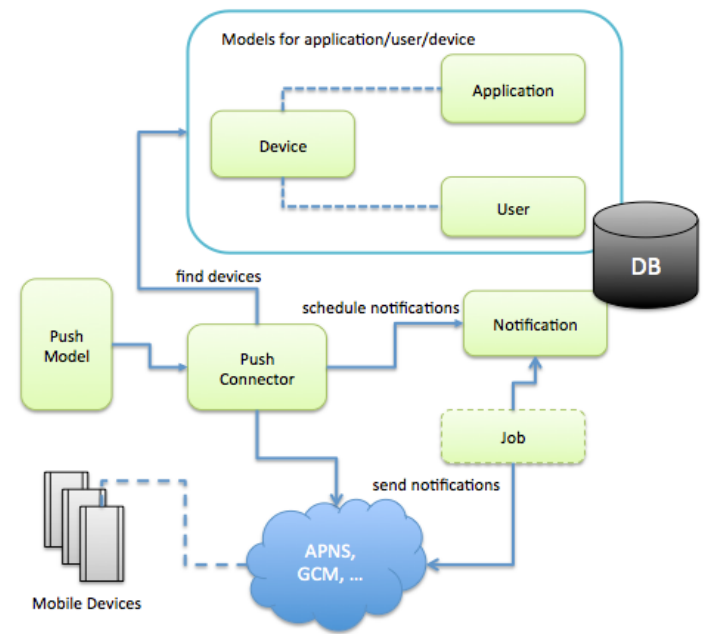

Figure 1: Architecture of Cloud to Device Messaging (C2DM) 
The components are:

- Device model and APIs to manage devices with applications and users.

- Application model to provide push settings for device types such as iOS and Android.

- Notification model to capture notification messages and persist scheduled notifications.

- Optional job to take scheduled notification requests.

- Push connector that interacts with device registration records and push providers APNS for iOS apps and GCM for Android apps.

Push model to provide high-level APIs for device-independent push notifications.

Advantages and Disadvantages of C2DM

Advantages:

- Using C2DM developers can develop more productive applications

- C2DM is connected to cloud so developers can get benefits of cloud computing in their applications

Disadvantages:

- Internet connectivity is required

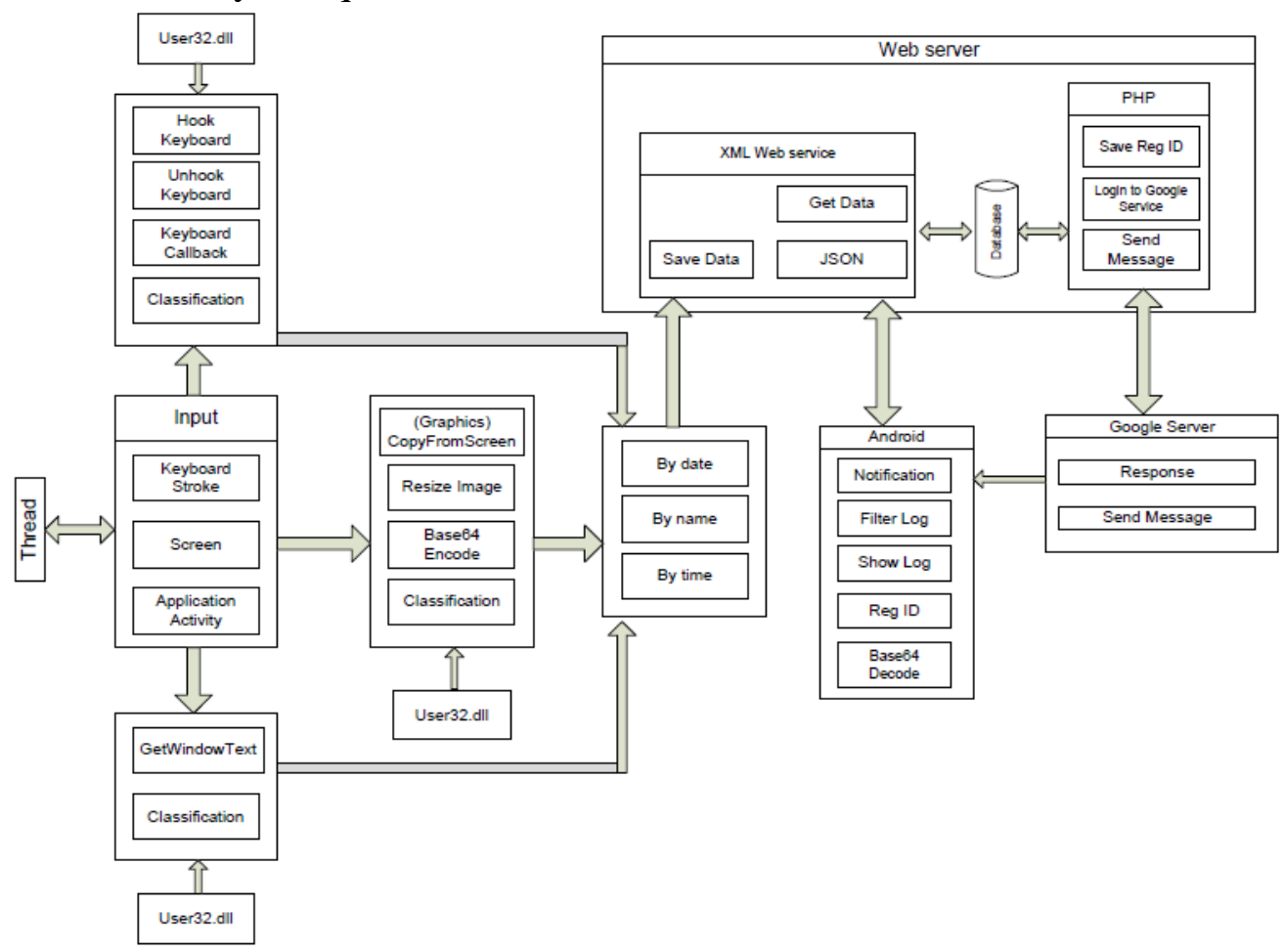

Figure 2: System Overview
- Limited message size

\section{Keylogger}

Keylogger is a program designed specifically to record all keyboard activity and save the results into a $\log$ or a text note. Keylogger type used in this system is a software keylogger application form that will be installed on a computer that is capable of recording all the input from the keyboard.

To be able to record all keyboard activity, keylogger should be run after the operating system is running and get in on the desktop display. Because keylogger is software that is watching, the keylogger will run in the background process and is not known by the user who uses the computer.

\section{RESULT}

In order to perform the features of this system all devices must be connected to the internet especially keylogger application, as based on a predetermined interval keylogger will automatically send a log or history to the web server to be stored. 


\section{Keylogger Implementation}

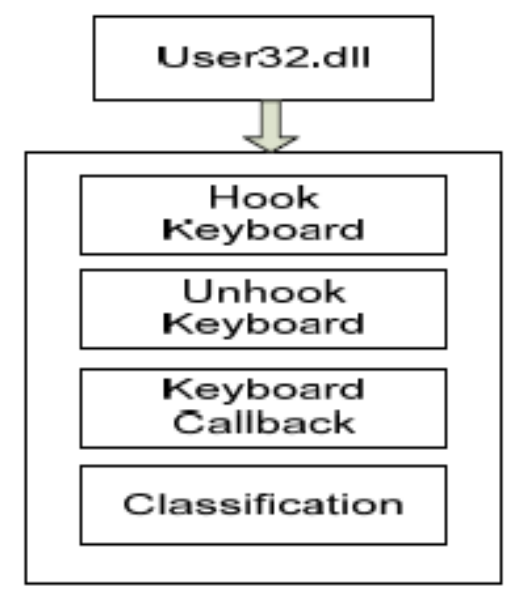

Figure 3. Keylogger Diagram

This process occurs done when the user press any button on keyboard, this incident arrested using HookKeyboard function() which is a Windows API from windows operating system, therefore, this process requires a library user32.dll already provided windows.

'create the hook

Public Sub HookKeyboard()

callback $=$ New

KeyboardHookDelegate(AddressOf

KeyboardCallback)

KeyboardHandle $=$

SetWindowsHookEx(13,callback,

process.GetCurrentProcess.MainModule.Bas eAddress, 0)

End Sub

Further functions HookKeyboard () will call each address on the keyboard previously shaped character ASCII Hex to convert into ASCII characters, because ASCII Hex number and difficult to read. Furthermore, the results of

the function HookKeyboard () will be classified based on the user account name, date, time, title applications, namely titles contained in each application running and $\operatorname{logs}$ of input keyboard that has been shaped the character string. The process of recording keyboard activity can be stopped by calling a function Unhookkeyboard ().

Public Sub UnhookKeyboard() If (Hooked()) Then If UnhookWindowsHookEx(Keyboard Handle)

$$
\begin{aligned}
& \text { <> } 0 \text { Then } \\
& \text { KeyboardHandle }=0
\end{aligned}
$$
End If

End If

End Sub

Cloud to Device Messaging (C2DM) Implementation 


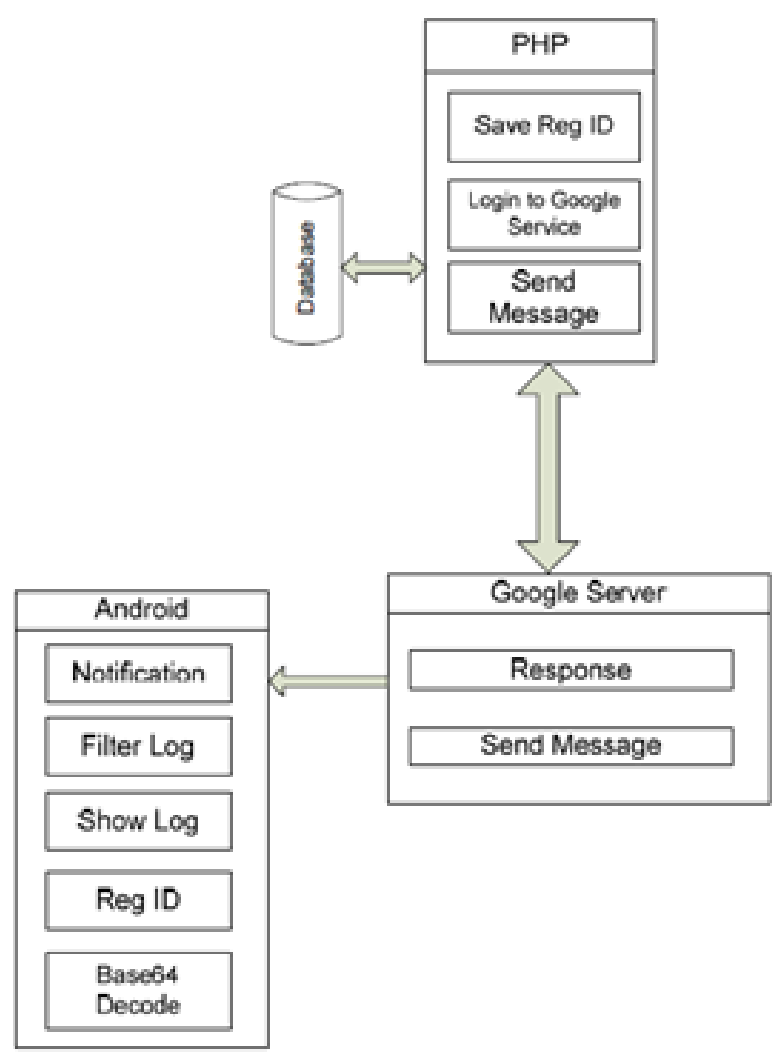

Figure 4. C2DM Diagram

\section{DISCUSSION}

The process of recording activity keyboard starts when there is an interaction of users who use a computer or referred to as user activity, user activity can include the activities of the occurs when the computer keyboard is pressed and form a screen capture of the screen the currently active application result in the form of log data, the process is carried out automatically by the application.
Furthermore, the application will add user information, data taken from the user account each computer. Next the data will be temporarily stored log data the local database before being sent to web server. The next process if local database is found, the data log will be stored temporarily on the table keystroke, but if the local database is not found then the log data is not will

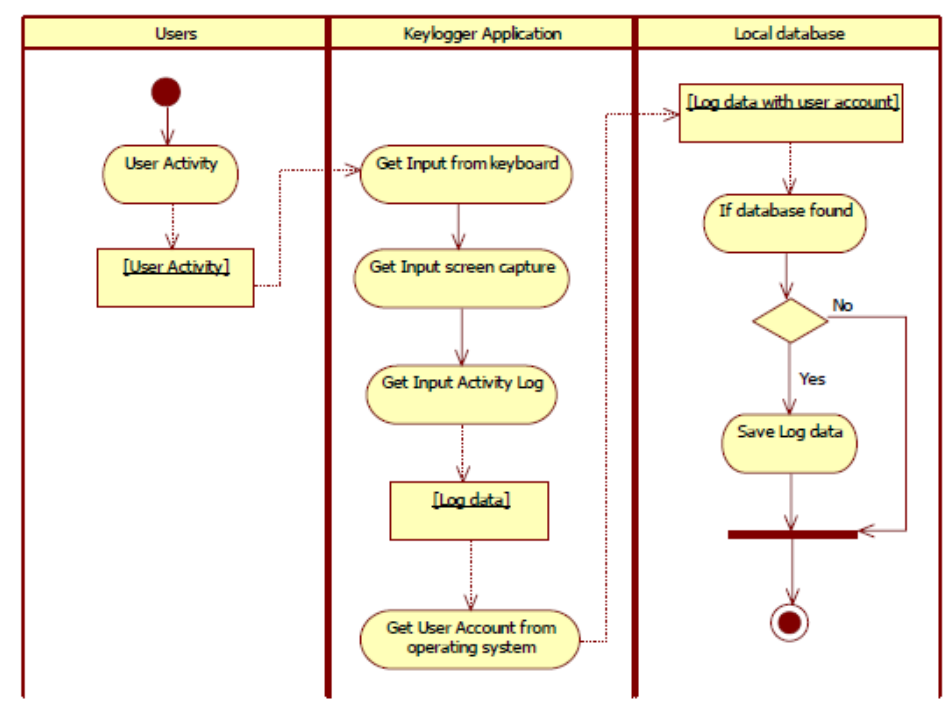

Figure 5. Record Activities Keyboard Activity Diagram 
The process starts from the log displays from a web server that has receive a new log of keylogger application will tell C2DM server that there is a new log must be notified on the android phone, android phone that receives the message will display the new log notifications on parents, the new log information is not overall log but only log header form dated and computer users. Leaders who wish to see more detailed log information can select the preview log on mobile applications, further requests will be

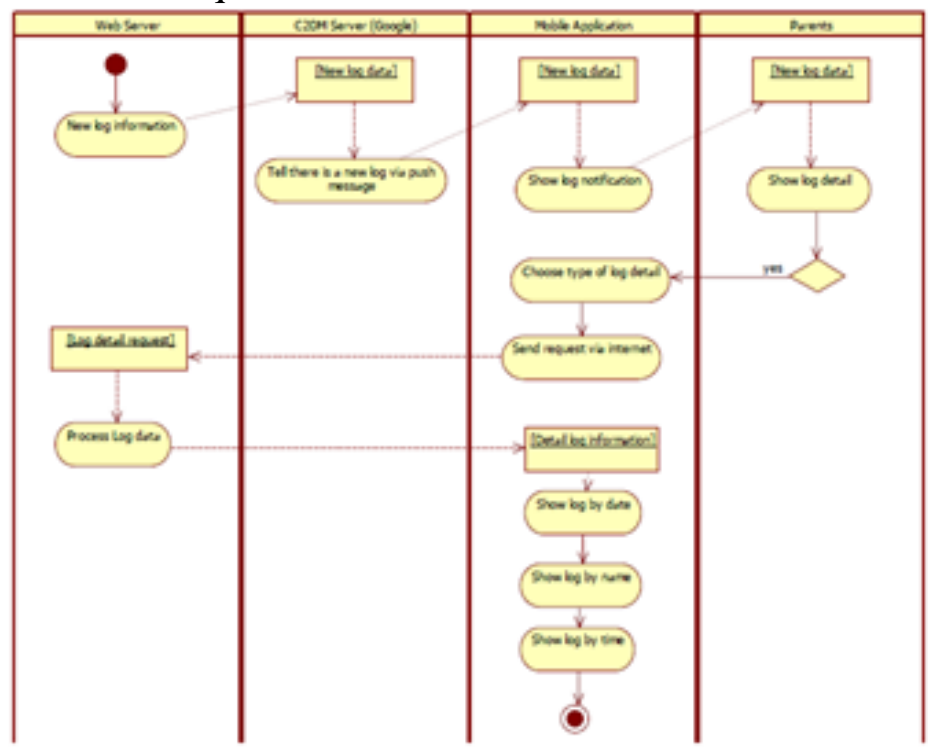

Figure 6. Activity Diagram

In addition to information such as message, the system uses the protocol http: // notification, the system also has the function search so that users can log search the log by date, name user and time, but the process

The log search is no longer use as the sender via the Internet to the requesting and receive data from the web server in XML and JSON formats. Here are the results $\log$ that appears on the phone.

\section{C2DM}

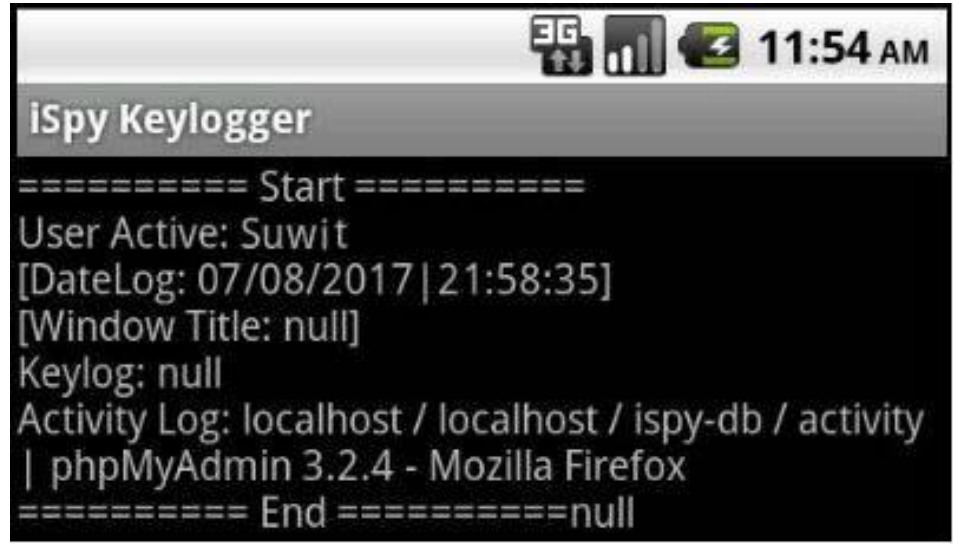

Figure 7. The results of the log on phone 


\section{CONCLUSION}

Based on the implementation and evaluation, can concluded:

1. With the push service message Android C2DM regulatory process activities of computer use can be done. With this system, the role of monitoring activity of computer not to be missed.

2. Integration process of keylogger, web server and mobile android successfully through internet communications network.
The conclusion that can be made after seeing a picture and description in previous chapters is to enable leaders in an organization such as a company to monitor the computer activity of his men anywhere and anytime. Suggestion for further research namely that this monitoring activity can be performed in real time. 


\section{REFERENCES}

Amin Bakhshandeh Abkenar, "Cloud-Hosted Continuous Activity Recognition Using OntologyBased Stream Reasoning “, 2nd IEEE International Conference, 2014.

Ashim Saha, Vimal Vijayakumar, Nirmalya Kar and Dwijen Rudrapal, "C2DMpush - An implementation of Android Cloud to Device Messaging Technology", IJITCS, vol 10, no. 3, 2013.

C. Paniagua, S. N. Srirama and H. Flores, "Bakabs: Man-aging Load of Cloud-Based Web Applications from Mo-biles," Proceedings of the 13th International Conference on Information Integration and Web-Based Applications and Services, ACM, New York, pp. 485-490, 2011.

J. Wijaya and K. Setiawan, "Monitoring Beberapa Komputer di Laboratorium UPH Surabaya," GEMA Aktual., vol. 4, no. 1, 2015.

R. Stannard, "Screen capture software for feedback in language education," Proc. Second Int. Wirel. Ready Symp., pp. 16-20, 2008.

S. Sagiroglu and G. Canbek, "Keyloggers," in IEEE Technology and Society Magazine, vol. 28, no. 3, pp. 10-17, 2009.

\section{BIOGRAPHY}

Suwitno received his Bachelor degree in Information System (S.Kom) from Buddhi Dharma University, Indonesia and Master Degree in Computer Sciences (M.Kom) concentration in Applied Computing Engineering from Budi Luhur University, Indonesia. He is a lecturer at the Department of Information Management, Faculty of Information Technology, Buddhi Dharma University.

Rino received his Bachelor degree in Information Technology (S.Kom) from Buddhi Dharma University, Indonesia and Master Degree in Computer Sciences (M.Kom) concentration in Software Engineering from Eresha University, Indonesia. He is a lecturer at the Department of Information System, Faculty of Information Technology, Buddhi Dharma University. 